\title{
Decreased Diastolic Ventricular Kinetic Energy in Young Patients with Fontan Circulation Demonstrated by Four-Dimensional Cardiac Magnetic Resonance Imaging
}

\author{
Pia Sjöberg ${ }^{1,5} \cdot$ Einar Heiberg $^{1,3,4} \cdot$ Pär Wingren ${ }^{5}$ Jens Ramgren Johansson ${ }^{2}$ • \\ Torsten Malm ${ }^{2} \cdot$ Håkan Arheden ${ }^{1,5} \cdot$ Petru Liuba $^{2} \cdot$ Marcus Carlsson $^{1,5}$
}

Received: 26 August 2016 / Accepted: 30 December 2016 / Published online: 10 February 2017

(c) The Author(s) 2017. This article is published with open access at Springerlink.com

\begin{abstract}
Four-dimensional (4D) flow magnetic resonance imaging (MRI) enables quantification of kinetic energy (KE) in intraventricular blood flow. This provides a novel way to understand the cardiovascular physiology of the Fontan circulation. In this study, we aimed to quantify the $\mathrm{KE}$ in functional single ventricles. 4D flow MRI was acquired in eleven patients with Fontan circulation (median age 12 years, range 3-29) and eight healthy volunteers (median age 26 years, range 23-36). Follow-up MRI after surgical or percutaneous intervention was performed in 3 patients. Intraventricular KE was calculated throughout the cardiac cycle and indexed to stroke volume (SV). The systolic/diastolic ratio of $\mathrm{KE}$ in Fontan patients was similar to the ratio of the controls' left ventricle (LV) or right ventricle (RV) depending on the patients' ventricular morphology (Cohen's $\kappa=1.0)$. Peak systolic KE/SV did not differ in patients compared to the $\mathrm{LV}$ in controls $(0.016 \pm 0.006 \mathrm{~mJ} /$
\end{abstract}

Electronic supplementary material The online version of this article (doi:10.1007/s00246-016-1565-6) contains supplementary material, which is available to authorized users.

Marcus Carlsson

marcus.carlsson@med.lu.se

1 Department of Clinical Sciences Lund, Clinical Physiology, Skane University Hospital, Lund University, Lund, Sweden

2 Department of Clinical Sciences Lund, Pediatric Heart Center, Skane University Hospital, Lund University, Lund, Sweden

3 Department of Biomedical Engineering, Faculty of Engineering, Lund University, Lund, Sweden

4 Center for Mathematics, Faculty of Engineering, Lund University, Lund, Sweden

5 Department of Medical Imaging and Physiology, Skane University Hospital, Lund, Sweden $\mathrm{ml}$ vs $0.020 \pm 0.004 \mathrm{~mJ} / \mathrm{ml}, p=0.09)$. Peak diastolic $\mathrm{KE} /$ $\mathrm{SV}$ in Fontan patients was lower than in the LV of the control group $(0.028 \pm 0.010 \mathrm{~mJ} / \mathrm{ml}$ vs $0.057 \pm 0.011 \mathrm{~mJ} /$ $\mathrm{ml}, p<0.0001)$. The KE during diastole showed a plateau in patients with aortopulmonary collaterals. This is to our knowledge the first study that quantifies the intraventricular $\mathrm{KE}$ of Fontan patients. KE is dependent on the morphology of the ventricle, and diastolic KE indexed to SV in patients is decreased compared to controls. The lower KE in Fontan patients may be a result of impaired ventricular filling.

Keywords Fontan - Congenital heart disease $\cdot$ Kinetic energy $\cdot \mathrm{CMR} \cdot \mathrm{MRI} \cdot \mathrm{Magnetic}$ resonance imaging

$\begin{array}{ll}\text { Abbreviations } \\ \text { APC } & \text { Aortopulmonary collaterals } \\ \text { ASD } & \text { Atrial septal defect } \\ \text { AVSD } & \text { Atrioventricular septal defect } \\ \text { BSA } & \text { Body surface area } \\ \text { CI } & \text { Cardiac index } \\ \text { CMR } & \text { Cardiac magnetic resonance } \\ \text { CO } & \text { Cardiac output } \\ \text { CoA } & \text { Coarctation of the aorta } \\ \text { DILV } & \text { Double inlet left ventricle } \\ \text { DORV } & \text { Double outlet right ventricle } \\ \text { EDVI } & \text { End-diastolic volume index } \\ \text { EF } & \text { Ejection fraction } \\ \text { ESVI } & \text { End-systolic volume index } \\ \text { HLHS } & \text { Hypoplastic left heart syndrome } \\ \text { IVC } & \text { Inferior vena cava } \\ \text { KE } & \text { Kinetic energy } \\ \text { LPA } & \text { Left pulmonary artery } \\ \text { LV } & \text { Left ventricle } \\ \text { MAPCA } & \text { Major aortopulmonary collateral arteries }\end{array}$




$\begin{array}{ll}\text { NYHA } & \begin{array}{l}\text { New York Heart Association Functional } \\ \text { Classification }\end{array} \\ \text { PLE } & \text { Protein-losing enteropathy } \\ \text { PS } & \text { Pulmonary stenosis } \\ \text { RV } & \text { Right ventricle } \\ \text { SV } & \text { Stroke volume } \\ \text { SVC } & \text { Superior vena cava } \\ \text { TAPVD } & \begin{array}{l}\text { Total anomalous pulmonary venous } \\ \text { drainage }\end{array} \\ \text { TGA } & \text { Transposition of the great arteries } \\ \text { VSD } & \text { Ventricular septal defect } \\ \text { 4D PC-MRI } & \begin{array}{l}\text { Four-dimensional phase-contrast magnetic } \\ \text { resonance imaging }\end{array}\end{array}$

\section{Introduction}

The Fontan operation is the procedure of choice used to palliate patients with a congenital heart defect not suited for biventricular repair [1]. One functional ventricle pumps the blood through the systemic circulation and the blood returns passively to the pulmonary circulation through the caval veins.

The Fontan circulation is dependent on an elevated systemic venous pressure, low pulmonary vascular resistance, and low left atrial pressure [2,3]. The patients are also dependent on a systemic ventricle with good systolic function, but since the primary limitation of the Fontan circulation is the filling of the systemic ventricle, a good diastolic function is mandatory for preserving an adequate cardiac output [4].

Studies show decrease in survival beyond 15-20 years after surgery in Fontan patients [5, 6] and the number of additional interventions after the Fontan procedure is high [7]. This is mainly driven by the prognosis of the Fontan patients who develop complications due to longstanding low cardiac output [8]. Increased understanding of the Fontan circulation physiology is therefore central to be able to prevent and treat possible complications.

The ability of the ventricle to deliver cardiac output to the body is facilitated by the looped shape anatomy of a morphological left ventricle that helps to conserve the momentum of blood [9] through a ventricular inflow vortex $[10,11]$. This means that the momentum of blood is preserved during diastole in a vortex and directed toward the outflow tract [11]. Four-dimensional (4D) phase-contrast cardiac magnetic resonance imaging (MRI) measures velocities of the blood in three directions over time and has been used to improve the accuracy in flow measurement over the atrioventricular valve in patients with Fontan circulation [12]. Furthermore, 4D flow enables quantification of caval flow distribution to the pulmonary artery [13]. 4D flow MRI can also quantify the kinetic energy (KE) of the heart chambers [14-19] and hence provide fundamental insights into these aspects of heart physiology. Studies in adult patients with heart failure and patients with mitral regurgitation have shown alterations in KE [20-22] and recently Jeong et al. used this method to quantify right ventricular KE in Tetralogy of Fallot patients [23]. Knowledge of KE in single ventricles and Fontan circulation may improve our understanding of the hemodynamic reasons for impaired function in these patients, but to our knowledge there are no published studies to date on ventricular KE data in these patients.

Therefore, the aim of this study was to noninvasively quantify the kinetic energy during the entire cardiac cycle of the single ventricle in Fontan patients using four-dimensional phase-contrast magnetic resonance imaging (4D PCMRI) to better understand the circulation and the pathophysiology of complications.

\section{Materials and Methods}

\section{Study Design}

Patients with Fontan circulation referred to Skane University Hospital for a cardiovascular magnetic resonance (CMR) examination were prospectively included. The inclusion time was from November 2013 until September 2014. In addition to routine clinical examination, 4D flow was acquired. Eight healthy volunteers ( 2 females, median age 26 years, range 23-36) were used as controls. Healthy controls were recruited by advertising at the local institution and had to have blood pressure $<140 / 90$, normal ECG, no cardiovascular medication, and no medical history of cardiovascular or other systemic disease to be included. The Fontan KE was compared on a group level to the LV $\mathrm{KE}$ of controls. The reason for comparing with the LV was that even if there is an RV morphology in a Fontan patient, the systemic ventricle is pumping with the requirements closer to an $\mathrm{LV}$ rather than an RV.

The principles of the Helsinki declaration were followed and the study was approved by the Regional Ethical Review Board, Lund, Sweden. Written informed consent was obtained from all subjects or their parents if $<18$ years of age.

\section{Magnetic Resonance Imaging}

4D PC-MRI flow acquisitions and cine images were acquired as previously described and validated in vivo and in vitro [24, 25]. In short, images were acquired using retrospective ECG triggering, with a 1.5 or $3 \mathrm{~T}$ Philips Achieva MRI. A volume covering the entire heart and central great vessels was acquired using a 4D phase-contrast 
velocity mapping turbo-field-echo sequence. Typical imaging parameters were TR/TE/flip angle: $6.2 \mathrm{~ms} / 3.6 \mathrm{~ms} / 8^{\circ}$ and velocity encoding $100 \mathrm{~cm} / \mathrm{s}$. Number of time phases acquired was dependent on heart rate and set to the maximum with a segmentation factor of 2 . The acquired temporal resolution varied from 50 to $55 \mathrm{~ms}$, i.e., 14-22 phases acquired and thereafter reconstructed to 40 time phases. Voxel size was $2 \times 2 \times 2 \mathrm{~mm}$ in children and $3 \times 3 \times 3 \mathrm{~mm}$ in adults. Acquired matrix size was typically $80 \times 80 \times 35$. Images were acquired during free breathing without navigator triggering as earlier described and validated [26].

A steady-state free precession sequence (TR/TE/flip angle: $2.9 \mathrm{~ms} / 1.5 \mathrm{~ms} / 60^{\circ}$, slice thickness $8 \mathrm{~mm}$, in-plane resolution $1.2 \times 1.2 \mathrm{~mm}$ ) was used to collect cine images covering the entire heart. Flow measurements with a phase-contrast velocity mapping fast field echo sequence (TR/TE/flip angle: $10 \mathrm{~ms} / 6.5 \mathrm{~ms} / 15^{\circ}$ in-plane resolution $1.2 \times 1.2 \mathrm{~mm}$ ) during free breathing as earlier described and validated were performed in the ascending aorta to measure the effective stroke volume (SV) and cardiac index (CI) [27].

Gadolinium contrast is not required for the 4D flow acquisitions and was not given as part of the study protocol but was administered in $2 / 11$ patients due to clinical questions of myocardial fibrosis in one case and MR angiography in one case.

\section{Image Analysis}

Images were analyzed with the Segment software (http:// segment.heiberg.se), using an in-house developed module for 4D PC-MRI analysis of KE [16]. A first-order polynomial fit to stationary tissue was used to compensate for eddy currents. Maxwell effects due to concomitant gradients were compensated for by the scanner. Velocity aliasing was corrected using phase unwrapping. The net threedimensional velocity vector for each voxel was computed.

Endocardial contours of the functional single ventricle and the contours of the superior vena cava (SVC), the tunnel or conduit from the inferior vena cava (IVC), and the pulmonary artery (PA) branches were manually traced in all phases of the cine images. The segmentation was imported to the reconstructed $4 \mathrm{D}$ flow dataset with manual correction when needed. KE was calculated within the resulting delineation of the ventricle throughout the cardiac cycle in the $4 \mathrm{D}$ flow images. The KE of a voxel was calculated as $\mathrm{KE}=1 / 2 m v^{2}$, where $m$ is the mass of the blood in the voxel and $v$ is the velocity of the voxel. The mass of the voxel was calculated as the volume multiplied by the density of the blood, $1.05 \mathrm{~g} / \mathrm{cm}^{3}$ [28]. The $\mathrm{KE}$ of each voxel within the delineation of the ventricle or vessel was summed to get the KE of the entire ventricle or vessel part in each phase of the cardiac cycle. To be able to compare patients with different age and body size, KE was indexed to stroke volume (SV) or the volume of the vessel part.

The location of the KE in the ventricle was performed by exporting velocity data and anatomical images from Segment to the open-source 4D flow software FourFlow (http://fourflow.heiberg.se) enabling anatomical correlation of KE. Peak systolic and diastolic velocities were quantified in the outflow and inflow of the ventricles, and correlated to systolic and diastolic peak KE, respectively. The electronic supplement file Movie 1 shows step by step how KE was analyzed using 4D flow MRI.

\section{Clinical Evaluation}

Patients' clinical status was evaluated by their treating pediatric cardiologist. Aortopulmonary collaterals (APCs) were evaluated during catheterization using fluoroscopic angiography of the thoracic aorta and the degree of collateral flow from MRI. APC blood flow was calculated using MRI by subtracting the flow volume in the caval veins from the aortic flow measured by 2D flow. The difference between pulmonary and caval venous flows was used for APC quantification in three patients where aortic flow measurements were not available [29]. Seven of the 11 patients underwent catheterization as part of the clinical evaluation. Hemodynamically significant APCs were defined as enlarged APC with saturation step-up in the pulmonary artery branches in combination with elevated end-diastolic pressure (measured by catheterization) and $>25 \%$ APC flow contribution to SV. Complications were defined as the need of intervention in the Fontan circulation.

\section{Statistical Analysis}

Statistical analysis was performed using GraphPad (v6.04, La Jolla, CA; USA). Ventricular KE is presented as means \pm SD. Differences in KE between Fontan patients and healthy volunteer groups were assessed using the Mann-Whitney test.

Since the number of patients in each group was small, statistical comparisons were not performed for differences between subgroups.

Cohen's kappa was analyzed for the relation of KE pattern and ventricular morphology.

\section{Results}

Eleven patients with Fontan circulation underwent 4D PCMRI. One example of intracardiac and cavopulmonary blood flow is shown in Movie 2 in electronic supplements. The patients' characteristics are shown in Table 1. 
Five clinically stable patients ( 2 female, median age 14, range 4-29) and 6 patients (1 female, median age 5 , range 3-15) who had complications were included in the study. Six patients had complications with need of intervention for pulmonary branch stenosis in four cases, restrictive atrial communication in one case, and severe APC in one case. Three patients underwent a follow-up MRI after surgical or percutaneous intervention. The interventions are described in Table 2. Five patients were below 10 years of age and these examinations were performed under general anesthesia.

\section{KE Pattern and Location in the Ventricle}

In all Fontan patients, two KE peaks were seen, one in systole and one in diastole. The KE throughout the cardiac cycle or the KE patterns is shown in Fig. 1. Three patients also had a late diastolic peak, corresponding to the atrial contraction, while the peaks during early filling phase

Table 1 Patient's characteristics

\begin{tabular}{|c|c|c|c|c|c|c|c|c|c|}
\hline Subject & $\begin{array}{l}\text { Age at time } \\
\text { of CMR (y) }\end{array}$ & Gender & $\mathrm{BSA}(\mathrm{m} 2)$ & $\begin{array}{l}\text { Ventricular mor- } \\
\text { phology }\end{array}$ & Type of Fontan & Diagnosis & APC & Complications & NYHA \\
\hline 1 & 3 & $\mathrm{M}$ & 0.56 & Left & Extracardiac & $\begin{array}{l}\text { Unbalanced AVSD, } \\
\text { small LV, PA, } \\
\text { MAPCA, right } \\
\text { isomerism, } \\
\text { bilateral SVC, } \\
\text { dextrocardia. } \\
\text { Fenestrated* }\end{array}$ & $*$ & PLE & NYHA II \\
\hline 2 & 7 & $\mathrm{M}$ & 0.82 & Left & Extracardiac & HLHS & Yes & Restrictive ASD & NYHA III \\
\hline 3 & 4 & M & 0.68 & Right & Extracardiac & $\begin{array}{c}\text { HLHS, TAPVD, } \\
\text { bilateral SVC }\end{array}$ & Yes & PLE & NYHA IV \\
\hline 4 & 12 & $\mathrm{~F}$ & 1.26 & Right & Extracardiac & $\begin{array}{l}\text { Hypoplastic right } \\
\text { PA, left isomer- } \\
\text { ism, interrupted } \\
\text { IVC, dextrocardia }\end{array}$ & No & $\begin{array}{l}\text { Pulmonary } \\
\text { arteriovenous } \\
\text { fistulas }\end{array}$ & NYHA IV \\
\hline 5 & 4 & M & 0.72 & Right & Extracardiac & $\begin{array}{l}\text { Hypoplastic aortic } \\
\text { arch, CoA, VSD, } \\
\text { DORV }\end{array}$ & Yes & LPA stenosis & NYHA II \\
\hline 6 & 15 & M & 1.60 & Right & Extracardiac & $\begin{array}{l}\text { DORV Taussig } \\
\text { Bing, unbal- } \\
\text { anced AVSD, PS, } \\
\text { TAPVD, right } \\
\text { isomerism }\end{array}$ & Yes & APC & NYHA II \\
\hline 7 & 17 & M & 1.72 & Right & Lateral tunnel & $\begin{array}{l}\text { Single ventricle, } \\
\text { DORV, TGA, } \\
\text { dextrocardia }\end{array}$ & No & No & NYHA I \\
\hline 8 & 14 & $\mathrm{~F}$ & 1.37 & Right & Extracardiac & $\begin{array}{l}\text { Single ventricle, } \\
\text { PS, TGA, right } \\
\text { isomerism, } \\
\text { bilateral SVC, } \\
\text { TAPVD }\end{array}$ & Yes & No & NYHA I \\
\hline 9 & 13 & $\mathrm{~F}$ & 1.36 & Left & Extracardiac & $\begin{array}{l}\text { TGA, multiple } \\
\text { VSD, hypoplastic } \\
\text { pulm arteries }\end{array}$ & Yes & No & NYHA I \\
\hline 10 & 29 & $\mathrm{M}$ & 1.72 & Left & Right atrium to PA & Tricuspid atresia & No & No & NYHA I \\
\hline 11 & 4 & $\mathrm{M}$ & 0.72 & $\begin{array}{r}\text { Left with long } \\
\text { outflow tract }\end{array}$ & Extracardiac & $\begin{array}{l}\text { DILV, TGA, PS, } \\
\text { left pulm artery } \\
\text { stenosis }\end{array}$ & No & No & NYHA II \\
\hline
\end{tabular}

$C M R$ cardiac magnetic resonance; BSA body surface area; $A P C$ aortopulmonary collaterals; NYHA New York Heart Association Functional Classification; $A V S D$ atrioventricular septal defect; $L V$ left ventricle; $M A P C A$ major aortopulmonary collateral arteries; $S V C$ superior vena cava; $H L H S$ hypoplastic left heart syndrome; TAPVD total anomalous pulmonary venous drainage; $I V C$ inferior vena cava; $C O A$ coarctation of aorta; $V S D$ ventricular septal defect; $D O R V$ double outlet right ventricle; $P S$ pulmonary stenosis; TGA transposition of the great arteries; DILV double inlet left ventricle; $P L E$ protein-losing enteropathy; $A S D$ atrial septal defect; $L P A$ left pulmonary artery

*Fenestrated extracardiac tunnel which makes the assessment of APC difficult 
Table 2 Characteristics and type of intervention in three patients who underwent cardiac magnetic resonance before and after intervention

\begin{tabular}{|c|c|c|c|c|}
\hline Subject & Diagnosis & Type of Fontan & Reason for intervention & Intervention \\
\hline 4 & $\begin{array}{l}\text { Hypoplastic right pulmonary artery, } \\
\text { left isomerism, interrupted IVC, } \\
\text { dextrocardia }\end{array}$ & Extracardiac & $\begin{array}{l}\text { Pulmonary arteriovenous fistula. } \\
\text { All liver drainage to one lung }\end{array}$ & $\begin{array}{l}\text { Extracardiac conduit was converted to a } \\
\text { Y-graft from the inferior vena cava to } \\
\text { both pulmonary branches separately }\end{array}$ \\
\hline 5 & $\begin{array}{l}\text { Hypoplastic aortic arch, CoA, VSD, } \\
\text { DORV }\end{array}$ & Extracardiac & Stenosis of left pulmonary artery & $\begin{array}{l}\text { Dilatation and stenting of left pulmonary } \\
\text { artery }\end{array}$ \\
\hline 6 & $\begin{array}{l}\text { DORV Taussig Bing, unbalanced } \\
\text { AVSD, PS, TAPVD, right isomerism }\end{array}$ & Extracardiac & APC & Coiling of collaterals \\
\hline
\end{tabular}

$I V C$ inferior vena cava; $C O A$ coarctation of aorta; $V S D$ ventricular septal defect; DORV Double outlet right ventricle; $A V S D$ atrioventricular septal defect; $P S$ pulmonary stenosis; TAPVD total anomalous pulmonary venous drainage; $A P C$ aortopulmonary collaterals

and atrial contraction were completely or partially fused in the remaining subjects. Patients with a left ventricular morphology with a short outflow tract $(n=4)$ had a lower systolic KE peak and a relatively higher diastolic peak (systole/diastole $<1$ ). Patients with right ventricular morphology and a long outflow tract $(n=6)$ had a higher systolic than diastolic peak (systole/diastole $>1$ ). One patient, number 11 , had a morphological left ventricle (LV) where the blood had to pass through a VSD via small RV to the aorta. A high diastolic peak, but even higher systolic peak, was seen in this patient (systole/diastole $>1$ ). Visualization showed that the $\mathrm{KE}$ in systole was mainly located in the outflow tract and during diastole from the mitral valve into the ventricle. $\mathrm{KE}$ in one patient is visualized as an example in Fig. 2. Cohen's kappa for ventricular morphology and KE pattern was 1.0. Six patients had hemodynamically significant aortopulmonary collaterals (APCs) with a mean flow of $35 \% \pm 9$. Patients with APC had a diastolic plateau of KE but this was only seen in one of the patients (Patient 11) without significant APC (Fig. 1). Patients without significant APC had a mean APC flow of $17 \% \pm 9$. Patient 11 had an APC flow of $6 \%$.

\section{KE Values}

No significant difference was seen in systolic peak KE/SV in the Fontan group $(0.036 \pm 0.018 \mathrm{~mJ} / \mathrm{ml}, n=11)$ compared to the $\mathrm{LV}$ in controls $(0.048 \pm 0.012 \mathrm{~mJ} / \mathrm{ml}, n=8$, $p=0.27$, Fig. 3a). The diastolic peak KE/SV in Fontan patients $(0.028 \pm 0.010 \mathrm{~mJ} / \mathrm{ml})$ was lower than in the $\mathrm{LV}$ of the control group $(0.057 \pm 0.011 \mathrm{~mJ} / \mathrm{ml}, \mathrm{p}<0.0001$, Fig. 3b).

Peak KE/SV in systole in stable patients was $0.036 \pm 0.016 \mathrm{~mJ} / \mathrm{ml}$ and in patients with complications $0.035 \pm 0.021 \mathrm{~mJ} / \mathrm{ml}$. Peak KE/SV in diastole for stable vs patients with complications were $0.027 \pm 0.007 \mathrm{~mJ} / \mathrm{ml}$ and $0.029 \pm 0.012 \mathrm{~mJ} / \mathrm{ml}$.

Table 3 and Fig. 3c-f show the KE values for Fontan patients and controls divided into subgroups based on ventricular morphology. Statistical comparisons could not be made due to the limited sample size when subdividing the group, but Fontan patients tended to show lower values compared to healthy volunteers.

No correlation was seen between ejection fraction and $\mathrm{KE} / \mathrm{SV}$ regarding mean $(r=-0.27 ; p=0.40)$, peak systolic $(r=-0.22 ; p=0.50)$, and peak diastolic values $(r=-0.13$; $p=0.70)$. There were no correlations between peak systolic velocity and peak systolic $\mathrm{KE}(r=0.36, p=0.27)$ or peak diastolic velocity and peak diastolic $\mathrm{KE}(r=0.11, p=0.75)$.

There was a significant correlation between mean $\mathrm{KE}$ and ventricular volumes in both patients and controls. In patients, $r=0.9, p=0.0008$ for EDV and $r=0.9, p=0.0004$ for end-systolic volume (ESV); and in controls $r=0.7$; $p=0.05$ for EDV and $r=0.8 ; p=0.02$ for ESV.

The KE patterns in patients after intervention compared to baseline MRI are shown in Fig. 4. In all patients, the KE patterns were similar before and after intervention, but in patients 4 and 6 there was a change in the shape of the diastolic curve. Patient 4 had a surgical conversion of the extra cardiac conduit to a Y-graft from the hepatic veins to each pulmonary artery branch to better distribute the hepatic blood flow to the lungs [30, 31]. Before intervention, the curve had two diastolic peaks, but after surgery the early diastolic peak was higher and fused with the late diastolic peak. Patient 6 had a fused diastolic curve with a plateau before intervention; after coiling of the APC, the curve showed two diastolic peaks.

Interobserver variability for EDV was $-1 \pm 12 \%$.

\section{KE in the Fontan Pathway and Pulmonary Branches}

$\mathrm{KE}$ in the tunnel or conduit from IVC could be quantified in all the patients. KE could not be analyzed in both PA branches in all patients and in one case in SVC due to stent artifacts or too short vessel. Mean KE in the tunnel/conduit $(n=11)$ was $0.0058 \pm 0.0045 \mathrm{~mJ} / \mathrm{ml}$; in SVC $(n=10)$ $0.0093 \pm 0.0023 \mathrm{~mJ} / \mathrm{ml}$; in left PA $(n=8) 0.018 \pm 0.009$; and in right $\mathrm{PA}(n=8) 0.015 \pm 0.006 \mathrm{~mJ} / \mathrm{ml}$. In $5 / 11$ patients, KE could be quantified in SVC, the tunnel/conduit from the IVC, and both PA branches. In this subgroup, 
Fig. 1 Kinetic energy in the ventricle during the cardiac cycle. Right ventricular (RV) morphology is shown in solid line. Left ventricular (LV) morphology is shown in broken line. The left column shows Fontan patients with complications. The right column shows patients with Fontan circulation without complications and a graph showing RV and LV of the control group. Patients with APC (aortopulmonary collaterals) are marked with a star. Patient 1 had a fenestrated extracardiac conduit which makes the assessment of APC difficult
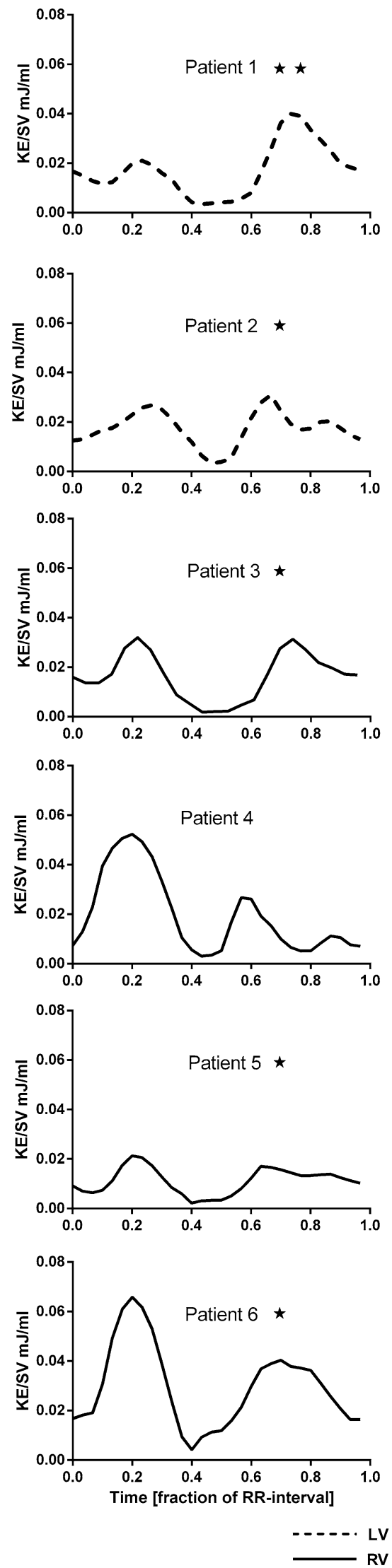
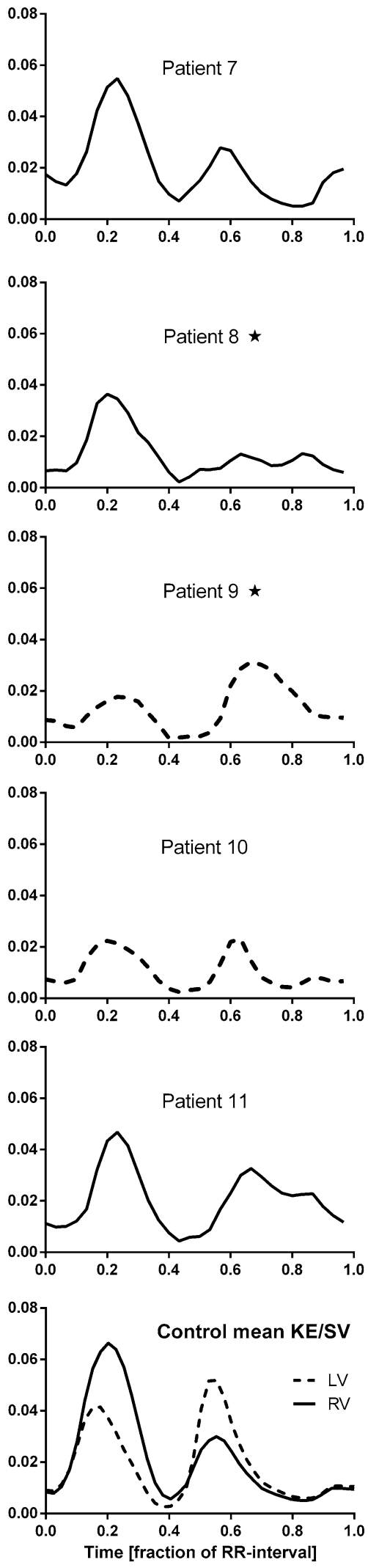

$\star$ APC

$\star \star$ Fenestration 
Fig. 2 Ventricular kinetic energy (KE) in a Fontan patient superimposed on CMR images to visualize the anatomical location of KE. The upper panel shows an oblique sagittal view in systole (a) and diastole (b). Systolic peak KE can be seen in a ventricular septal defect leading the blood to the aorta. Diastolic peak KE is located from the atrioventricular valve into the ventricle. The lower panel visualizes $\mathrm{KE}$ in an oblique transversal view in the same patient in systole (c) and diastole (d). AAo ascending aorta; $V S D$ ventricular septal defect; $V$ ventricle; $A$ atrium; $A V$ valve atrioventricular valve; AoV aortic valve; DAo descending aorta; $T$ lateral tunnel
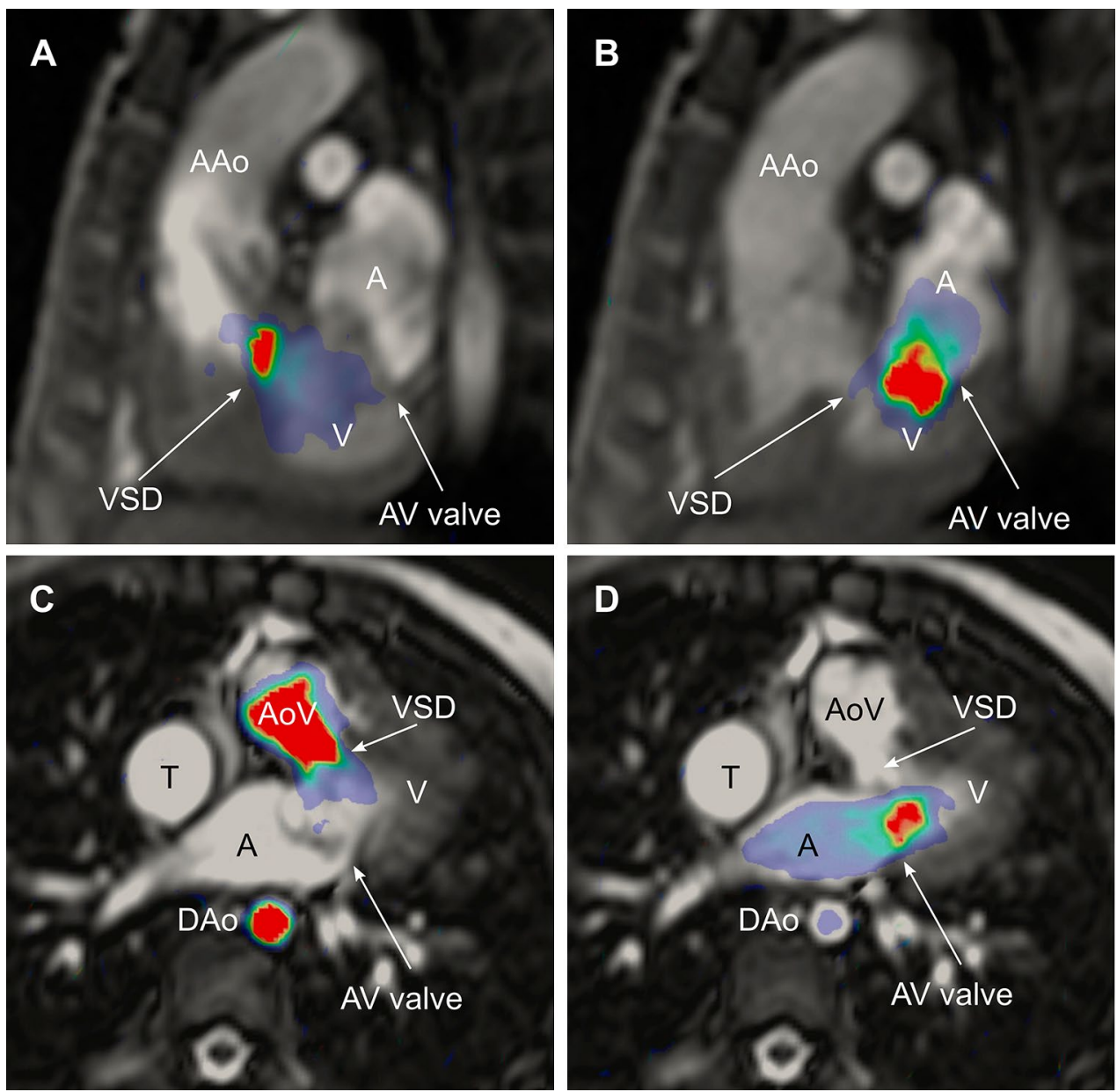

the mean KE was significantly higher in both PA branches together, $0.034 \pm 0.006$, than in the tunnel/conduit $+\mathrm{SVC}$, $0.014 \pm 0.006(p=0.008)$. The mean cross-sectional area of both PA branches together was $2.2 \pm 1.2 \mathrm{~cm}^{2}$ and in the tun$\mathrm{nel} /$ conduit $+\mathrm{SVC} 4.4 \pm 0.9 \mathrm{~cm}^{2}$.

\section{Discussion}

This study shows that kinetic energy of blood in the single ventricle during the cardiac cycle in Fontan patients is dependent on the left or right morphology of the ventricle and that peak diastolic KE is lower in patients with Fontan circulation compared to controls.

Diastolic KE was larger than systolic KE in patients with LV morphology similar to the LV of healthy volunteers. In patients with an RV morphology, the KE pattern was similar to that of the RV in healthy volunteers with a higher systolic KE compared to diastolic KE. The mechanisms for these differences may be that during systole, blood flows with higher velocities within a longer right ventricular outflow tract compared to the shorter left ventricular outflow tract, resulting in higher $\mathrm{KE}$ within the heart on the right side compared to the left.

During diastole, the blood is accelerated into the left ventricle primarily by ventricular recoil leading to high KE. This is in contrast to the right ventricle where filling of the ventricle is mainly caused by movement of the atrioventricular plane toward the base of the heart over the blood in the atrium which requires less $\mathrm{KE}$ as the blood does not need to be accelerated into the ventricle(9). The interventricular difference of the LV as a pressure pump and the RV as a volume pump [15] is reflected in the findings that LV mass is an independent predictor of LV diastolic KE and $\mathrm{RV}$ end-diastolic volume an independent predictor of RV diastolic KE [19].

The study also shows that peak diastolic KE/SV is lower in patients with Fontan circulation compared to controls. These findings differ from results in adult heart failure patients [32] where diastolic KE indexed to stroke volume was increased compared to controls. The explanation for a lower KE in Fontan patients compared to patients with congestive heart failure may be that the impaired ventricular filling and the forward failure problem in Fontan patients result in intracardiac flow with lower velocities and 
Fig. 3 Kinetic energy (KE) indexed to stroke volume. The left column shows peak KE during systole and the right column peak KE during diastole. a and b shows all Fontan patients and the left ventricle in the control group. $\mathbf{c}$ and $\mathbf{d}$ shows Fontan patients with LV morphology and the left ventricle in the control group. e and $\mathbf{f}$ shows Fontan patients with RV morphology and the right ventricle in the control group. Error bars show mean $\pm \mathrm{SD}$
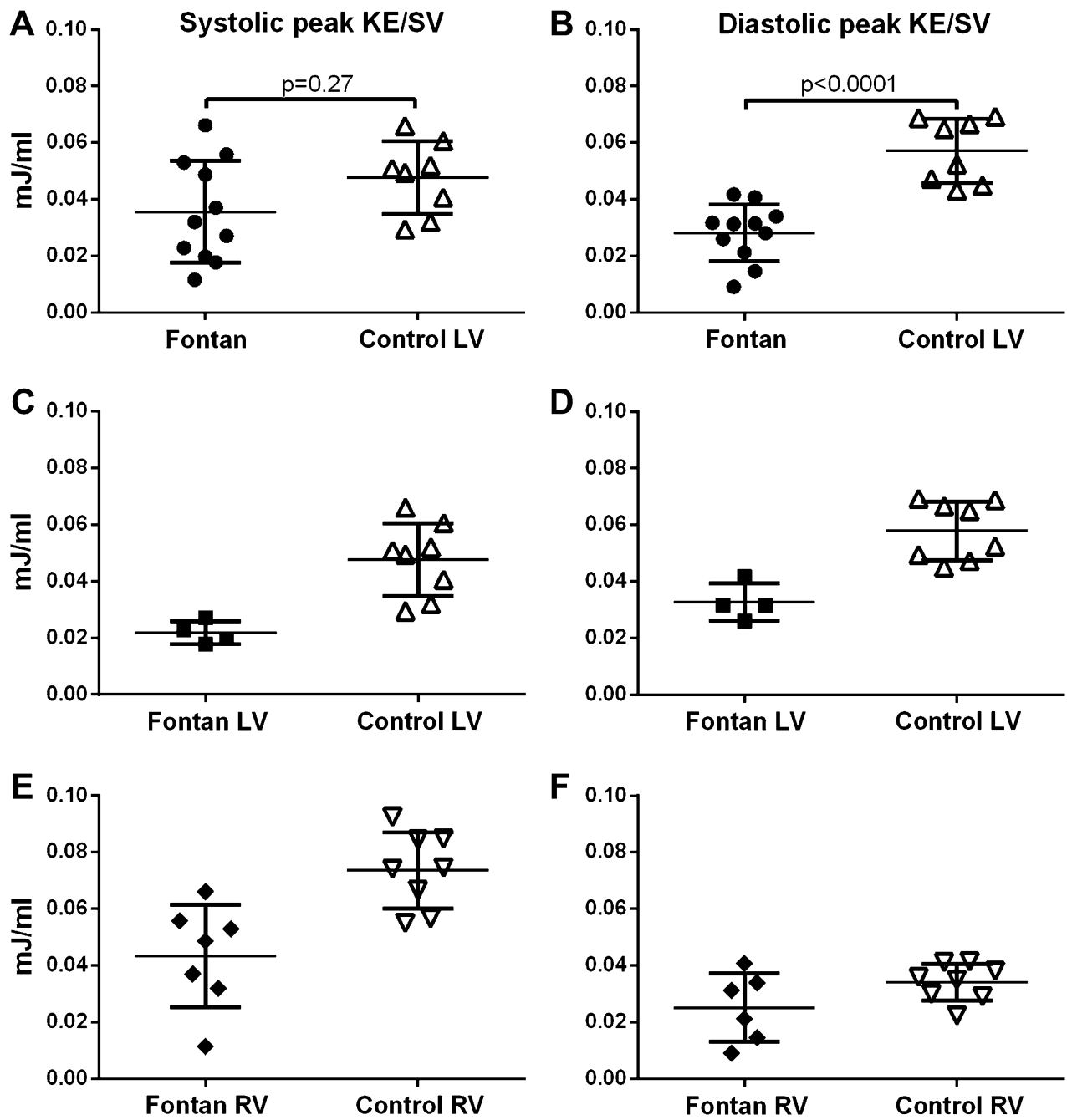

that this is represented as a lower KE. This is in contrast to adult heart failure which is characterized by predominant backward failure and increased filling pressures and increased $\mathrm{KE} / \mathrm{SV}$, independent of etiology being ischemic or nonischemic cardiomyopathy. A contributing factor to the difference could be that 7 out of 11 patients had right ventricle morphology and thereby low peak diastolic KE, but the difference seems to remain when comparing Fontan patients with left ventricle morphology with left ventricles of controls, although the number of patients are low. The fact that the majority of Fontan patients had RV morphology also explains why there was no significant difference in peak KE/SV in systole when comparing the Fontan group with the LV of controls. However, the results indicate that the systolic KE will differ between patients and controls when ventricular morphology is taken into account. We did not find any difference in KE between stable patients and patients with complications, and this may also be explained by the larger effect of ventricular morphology on KE.
The high correlation between KE and ventricular volumes is explained by the fact that $\mathrm{KE}$ is calculated from both velocity and mass of the blood. The large differences in size and heart rates in the study population were the reason to index KE to stroke volume (SV). SV was chosen as more appropriate compared to ventricular volumes as EDV differs greatly with the type of heart defect in Fontan patients. Stroke volume is the net result of ventricular filling and contraction and as such intuitive for indexing. Another way to index could be to body surface area as in, for example, Jeong et al.'s article on patients with tetralogy of Fallot [23]. Kinetic energy could also be indexed to cardiac index, since this indexation has been used in other studies, but this will create a difference between pediatric and adult hearts as the cardiac index is similar but the mass of blood is much smaller in the small heart leading to lower KE. Therefore, we indexed to stroke volume to be able to compare small and large hearts. 
Table 3 Kinetic energy indexed to stroke volume, body surface area, and cardiac index in Fontan patients compared to healthy controls, divided into type based on ventricular morphology

\begin{tabular}{|c|c|c|c|}
\hline Ventricular morphology & & Fontan patients & Controls \\
\hline \multirow{11}{*}{$\begin{array}{l}\text { Left ventricle } \\
\text { Fontan, } n=4 \\
\text { Control, } n=8\end{array}$} & EDVI, $\mathrm{ml} / \mathrm{m}^{2}$ & $102 \pm 42$ & $101 \pm 11$ \\
\hline & ESVI, $\mathrm{ml} / \mathrm{m}^{2}$ & $60 \pm 29$ & $43 \pm 8$ \\
\hline & $\mathrm{EF}, \%$ & $43 \pm 6$ & $57 \pm 5$ \\
\hline & $\mathrm{CO}, 1 / \mathrm{min}$ & $3.7 \pm 2.4$ & $6.5 \pm 1.2$ \\
\hline & $\mathrm{CI}, 1 / \mathrm{min} / \mathrm{m}^{2}$ & $4.0 \pm 1.5$ & $3.3 \pm 0.4$ \\
\hline & Peak systolic KE/SV, mJ/ml & $0.022 \pm 0.004$ & $0.048 \pm 0.012$ \\
\hline & Peak diastolic KE/SV, mJ/ml & $0.033 \pm 0.007$ & $0.058 \pm 0.010$ \\
\hline & Peak systolic KE/BSA, mJ/m² & $0.56 \pm 0.20$ & $2.57 \pm 0.60$ \\
\hline & Peak diastolic KE/BSA, mJ/m² & $1.42 \pm 0.73$ & $3.18 \pm 0.62$ \\
\hline & Peak systolic KE/CI, mJ/(1/min/m²) & $0.37 \pm 0.18$ & $1.55 \pm 0.38$ \\
\hline & Peak diastolic KE/CI, $\mathrm{mJ} /\left(1 / \mathrm{min} / \mathrm{m}^{2}\right)$ & $0.51 \pm 0.15$ & $1.93 \pm 0.48$ \\
\hline \multirow{11}{*}{$\begin{array}{l}\text { Right ventricle } \\
\text { Fontan, } n=7 \\
\text { Controls, } n=8\end{array}$} & EDVI, $\mathrm{ml} / \mathrm{m}^{2}$ & $87 \pm 20$ & $96 \pm 17$ \\
\hline & ESVI, $\mathrm{ml} / \mathrm{m}^{2}$ & $52 \pm 13$ & $40 \pm 10$ \\
\hline & $\mathrm{EF}, \%$ & $39 \pm 7$ & $59 \pm 6$ \\
\hline & $\mathrm{CO}, 1 / \mathrm{min}$ & $3.4 \pm 2.0$ & $6.8 \pm 1.5$ \\
\hline & $\mathrm{CI}, 1 / \mathrm{min} / \mathrm{m}^{2}$ & $2.9 \pm 1.0$ & $3.4 \pm 0.5$ \\
\hline & Peak systolic KE/SV, mJ/ml & $0.043 \pm 0.018$ & $0.074 \pm 0.014$ \\
\hline & Peak diastolic KE/SV, mJ/ml & $0.025 \pm 0.012$ & $0.034 \pm 0.007$ \\
\hline & Peak systolic KE/BSA, mJ $/ \mathrm{m}^{2}$ & $0.78 \pm 0.51$ & $4.04 \pm 0.87$ \\
\hline & Peak diastolic KE/BSA, mJ/m² & $0.96 \pm 0.54$ & $1.94 \pm 0.70$ \\
\hline & Peak systolic KE/CI, $\mathrm{mJ} /\left(1 / \mathrm{min} / \mathrm{m}^{2}\right)$ & $0.79 \pm 0.58$ & $2.36 \pm 0.65$ \\
\hline & Peak diastolic KE/CI, $\mathrm{mJ} /\left(1 / \mathrm{min} / \mathrm{m}^{2}\right)$ & $0.44 \pm 0.31$ & $1.11 \pm 0.38$ \\
\hline
\end{tabular}

$R V$ right ventricle; $L V$ left ventricle; $E D V I$ end-diastolic volume index; $E S V I$ end-systolic volume index; $E F$ ejection fraction; $C O$ cardiac output; $C I$ cardiac index; $K E$ kinetic energy; $S V$ stroke volume; $B S A$ body surface area; $C I$ cardiac index
Three patients in this study underwent a second examination after surgical or percutaneous intervention. The results show remarkably similar magnitude and basic pattern of KE over the cardiac cycle at the two examinations. This suggests that the KE pattern is quite specific to each patient. A possible explanation to the change in diastolic curve shape after intervention in patient 6 could be that the aortopulmonary collaterals before intervention caused an extra inflow with high pressure in the atrium leading to a plateau of the KE curve during diastole. This explanation is supported by the fact that all patients in this study who had clinically significant aortopulmonary collaterals (APCs) also had a plateau in the diastolic part of the $\mathrm{KE}$ curve. The rise in diastolic KE in patient 4 after surgery might reflect an increase in pulmonary arteriovenous fistulas coherent with the clinical picture. These results address the complexity in evaluating KE in patients with Fontan circulation and indicate that the clinical implication for using $\mathrm{KE}$ in this patient group primarily is to better understand the physiology and to follow the patients on an individual basis. In our study, only one patient had fenestration between the conduit and the atria. The possible effects on ventricular KE from flow through the fenestration are not known but it may be similar to APC with a diastolic plateau and higher KE in diastole if the fenestration causes higher preload. In one of the patients in whom no significant APC could be identified, there was a diastolic plateau. Larger numbers of Fontan patients with KE are needed to see if the diastolic plateau pattern is related to other clinical parameters than APC flow. Recently, Schiavazzi et al. used modeling to calculate the effects of left pulmonary artery stenosis on hemodynamics in single ventricles stage II surgery and found that the stenosis needed to be quite severe, $>65 \%$ of the diameter, to be hemodynamically significant [33]. This is in line with the findings of unchanged ventricular KE pattern before and after stenting of a left pulmonary stenosis in one of our study patients.

The analysis of KE indexed for stroke volume in the Fontan pathway showed a significant increase in KE from the caval veins to the PA branches. This is probably explained by the smaller cross-sectional area of the PA branches compared to the caval veins.

Another way to analyze $\mathrm{KE}$ in the ventricle is to divide it based on whether the volume goes directly from the atria to the aorta in the same heart beat or if it stays one or more heart beats in the ventricle [34]. Eriksson et al. [21] have suggested that KE might reflect ventricular dysfunction at an early stage. They found altered diastolic flow routes 

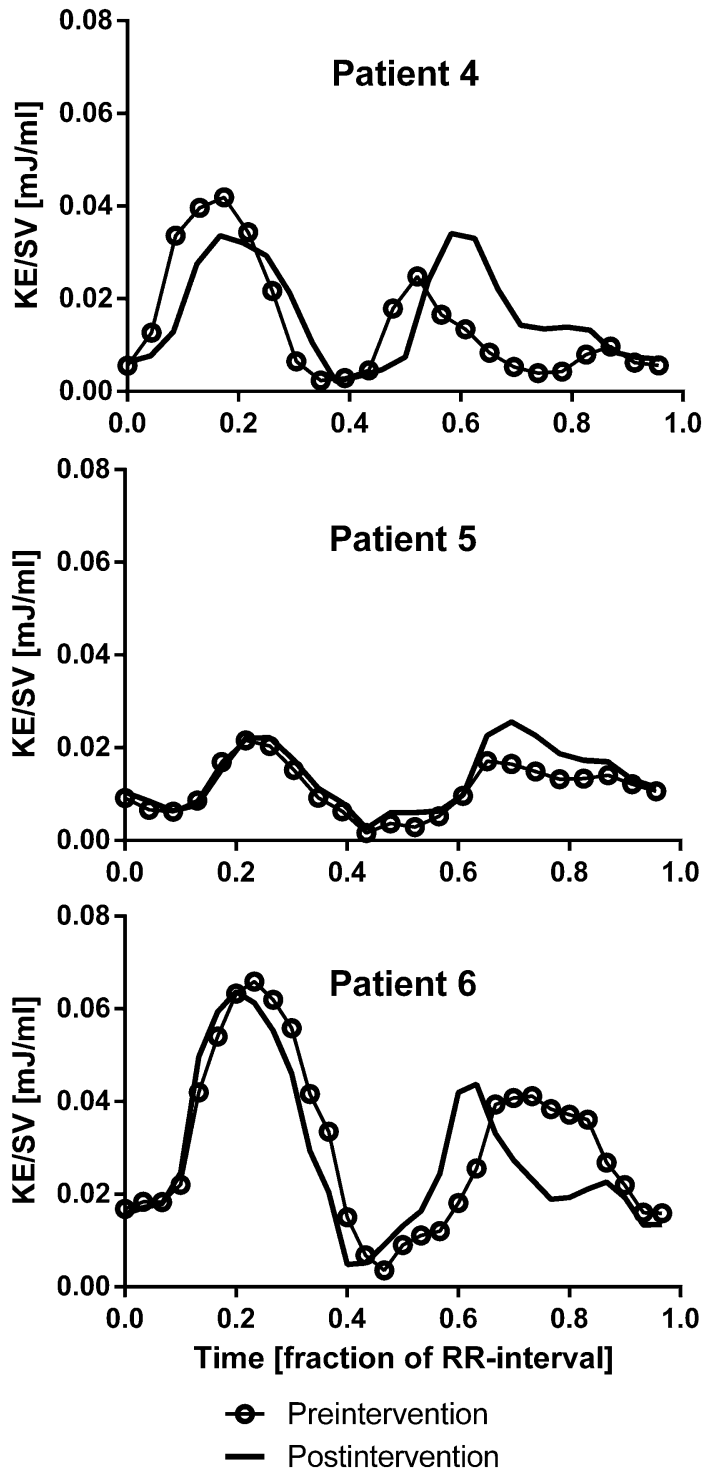

Fig. $4 \mathrm{KE}$ throughout the cardiac cycle in three patients before and after surgical or percutaneous intervention. Details of the intervention are listed in Table 2. Heart rate (beats/minute) pre-intervention/ post-intervention was in patient $4: 103 / 87$, patient $5: 85 / 83$, patient 6 : $63 / 80$

and impaired preservation of $\mathrm{KE}$ in late diastole in the $\mathrm{LV}$ of patients with dilated cardiomyopathy compared with healthy subjects. The changes in flow route and energetics were seen despite clinical compensation and might be useful as subclinical markers of LV dysfunction. Future studies using this analysis will provide information if this will give additional insights into the Fontan physiology. 4D flow has also been used to quantify kinetic energy of turbulent flow and this energy may be lost to heat [20]. In Fontan patients with slower velocities, turbulent flow is less likely and we did not find any patients with signal drop in the magnitude images which would have indicated turbulent flow.
A study of kinetic energy in patients with Tetralogy of Fallot, recently published by Jeong et al [23], shows that patients had a tendency to higher systolic peak KE compared to healthy volunteers and that a change in ventricular KE may prove to be an indication of ventricular dysfunction. Together with these studies and the results from studies in adult patients with heart failure [32], this study indicates that $\mathrm{KE}$ might be a useful noninvasive method of monitoring patients and detect signs of ventricular and circulatory dysfunction [35]. The finding that there is no correlation between KE and peak velocity shows that quantifying the entire velocity field in three dimensions of the entire ventricle has additional information compared to peak velocities. Kinetic energy as a single measure will probably not predict subsequent ventricular function but the future use will be in the context of several hemodynamic measures.

In Fontan patients, breathing contributes to the return of blood to the lungs to a higher extent than in normal physiology [36]. Therefore, we acquired flow measurements during free breathing and not respiratory triggered to endinspiration or end-expiration, to be able to get mean flow throughout the respiratory cycle.

\section{Study Limitations}

The study population is small and therefore statistical comparisons of the subgroups of left and right ventricular morphology and patients before and after intervention could not be made. Conclusions of the effect on KE of APC's or interventions are also limited by the small sample size, but the data presented can point to applications where future studies may provide insights of Fontan physiology. In the Fontan group, five out of eleven patients were ventilated during the examination and this may affect the results. However, the KE results from the ventilated patients do not appear different from the nonventilated patients, but this needs to be further investigated. The patient who was under anesthesia pre-intervention $(n=1)$ was also under anesthesia at the post-intervention CMR. Heart rates varied between examinations, but indexed KE remained similar independent of heart rate. There may be age-related differences in KE and in this study we did not have age matched controls. However, this was compensated for by indexing $\mathrm{KE}$ to $\mathrm{SV}$.

\section{Conclusion}

This is to our knowledge the first study that quantifies the intraventricular KE of Fontan patients. KE might be a useful noninvasive method of monitoring Fontan patients and 
detect signs of ventricular and circulatory dysfunction. Further studies are needed to determine if changes in ventricular KE can provide early information on ventricular dysfunction or for example the significance of aortopulmonary collaterals and guide medical and surgical intervention.

Acknowledgements Annika Maxedius is gratefully acknowledged for help with patient administration. Ann-Helen Arvidsson, Christel Carlander, and Håkan Månsson are gratefully acknowledged for MRI acquisitions.

Funding This work was funded by Swedish Heart-Lung foundation, Lund University; Skane University Hospital, Region of Skane; Swedish Research Council and Swedish Medical Association.

\section{Compliance with Ethical Standards}

Conflict of interest Einar Heiberg is stockholder and founder of the medical image analysis company Medviso AB that sells the Segment software for clinical use. Hakan Arheden is stockholder of Imacor AB, a core lab for medical image analysis. Marcus Carlsson has received consultancy fees from Imacor AB. The remaining authors have no disclosures.

Open Access This article is distributed under the terms of the Creative Commons Attribution 4.0 International License (http:// creativecommons.org/licenses/by/4.0/), which permits unrestricted use, distribution, and reproduction in any medium, provided you give appropriate credit to the original author(s) and the source, provide a link to the Creative Commons license, and indicate if changes were made.

\section{References}

1. Fontan F, Baudet E (1971) Surgical repair of tricuspid atresia. Thorax 26:240-248

2. Kurishima C, Saiki H, Masutani S, Senzaki H (2015) Tailored therapy for aggressive dilatation of systemic veins and arteries may result in improved long-term Fontan circulation. J Thorac Cardiovasc Surg 150:1367-1370

3. Abdulla R (2014) The Fontan procedure: now what? Pediatr Cardiol 35:1307-1308

4. Gewillig M, Goldberg DJ (2014) Failure of the fontan circulation. Heart Fail Clin 10:105-116

5. Khairy P, Fernandes SM, Mayer JE et al (2008) Long-term survival, modes of death, and predictors of mortality in patients with Fontan surgery. Circulation 117:85-92

6. Dabal RJ, Kirklin JK, Kukreja M et al (2014) The modern Fontan operation shows no increase in mortality out to 20 years: a new paradigm. J Thorac Cardiovasc Surg 148:2517-2524

7. Van Dorn CS, Menon SC, Johnson JT et al (2015) Lifetime cardiac reinterventions following the Fontan procedure. Pediatr Cardiol 36:329-334

8. d'Udekem Y, Iyengar AJ, Cochrane AD, et al (2007) The Fontan procedure: contemporary techniques have improved long-term outcomes. Circulation 116:I-157-I-164.

9. Kilner PJ, Yang GZ, Wilkes AJ et al (2000) Asymmetric redirection of flow through the heart. Nature 404:759-761

10. Töger J, Kanski M, Carlsson M et al (2012) Vortex ring formation in the left ventricle of the heart: analysis by $4 \mathrm{D}$ flow MRI and Lagrangian coherent structures. Ann Biomed Eng 40:1-11
11. Arvidsson PM, Kovács SJ, Töger J, et al (2016) Vortex ring behavior provides the epigenetic blueprint for the human heart. Sci Rep 6:22021.

12. She HL, Roest AAW, Calkoen EE et al (2016) Comparative Evaluation of Flow Quantification across the Atrioventricular Valve in Patients with Functional Univentricular Heart after Fontan's Surgery and Healthy Controls: Measurement by 4D Flow Magnetic Resonance Imaging and Streamline Visualization. Congenit Heart Dis

13. Bächler P, Valverde I, Pinochet N et al (2013) Caval blood flow distribution in patients with Fontan circulation: quantification by using particle traces from 4D flow MR imaging. Radiology 267:67-75

14. Arvidsson PM, Töger J, Heiberg E et al (2013) Quantification of left and right atrial kinetic energy using four-dimensional intracardiac magnetic resonance imaging flow measurements. J Appl Physiol 114:1472-1481

15. Carlsson M, Heiberg E, Toger J, Arheden H (2012) Quantification of left and right ventricular kinetic energy using four-dimensional intracardiac magnetic resonance imaging flow measurements. Am J Physiol Heart Circ Physiol 302:H893-H900

16. Heiberg E, Sjögren J, Ugander M et al (2010) Design and validation of Segment-freely available software for cardiovascular image analysis. BMC Med Imaging 10:1

17. Eriksson J, Dyverfeldt P, Engvall J et al (2011) Quantification of presystolic blood flow organization and energetics in the human left ventricle. Am J Physiol Heart Circ Physiol 300:H2135-H2141

18. Fredriksson AG, Zajac J, Eriksson J et al (2011) 4-D blood flow in the human right ventricle. Am J Physiol Heart Circ Physiol 301:H2344-H2350

19. Steding-Ehrenborg K, Arvidsson PM, Töger J, et al (2015) Determinants of kinetic energy of blood flow in the four-chambered heart in athletes and sedentary controls. Am J Physiol Heart Circ Physiol 310:113-122

20. Zajac J, Eriksson J, Dyverfeldt P et al (2015) Turbulent kinetic energy in normal and myopathic left ventricles. J Magn Reson Imaging 41:1021-1029

21. Eriksson J, Bolger AF, Ebbers T, Carlhäll C-JJ (2013) Fourdimensional blood flow-specific markers of LV dysfunction in dilated cardiomyopathy. Eur Heart $\mathbf{J}$ Cardiovasc Imaging 14:417-424

22. Al-Wakeel N, Fernandes JF, Amiri A et al (2015) Hemodynamic and energetic aspects of the left ventricle in patients with mitral regurgitation before and after mitral valve surgery. J Magn Reson Imaging 42:1705-1712

23. Jeong D, Anagnostopoulos PV, Roldan-Alzate A et al (2014) Ventricular kinetic energy may provide a novel noninvasive way to assess ventricular performance in patients with repaired tetralogy of Fallot. J Thorac Cardiovasc Surg 149:1339-1347

24. Töger J, Bidhult S, Revstedt J et al (2016) Independent validation of four-dimensional flow MR velocities and vortex ring volume using particle imaging velocimetry and planar laser-Induced fluorescence. Magn Reson Med 75:1064-1075

25. Carlsson M, Töger J, Kanski M et al (2011) Quantification and visualization of cardiovascular 4D velocity mapping accelerated with parallel imaging or k-t BLAST: head to head comparison and validation at $1.5 \mathrm{~T}$ and $3 \mathrm{~T}$. J Cardiovasc Magn Reson 13:55

26. Kanski M, Töger J, Steding-Ehrenborg K et al (2015) Wholeheart four-dimensional flow can be acquired with preserved quality without respiratory gating, facilitating clinical use: a head-tohead comparison. BMC Med Imaging 15:20

27. Carlsson M, Andersson R, Bloch KM et al (2012) Cardiac output and cardiac index measured with cardiovascular magnetic resonance in healthy subjects, elite athletes and patients with congestive heart failure. J Cardiovasc Magn Reson 14:51 
28. Trudnowski RJ, Rico RC (1974) Specific gravity of blood and plasma at 4 and 37 C. Clin Chem 20:615-616

29. Pushparajah K, Wong JK, Bellsham-Revell HR et al (2015) Magnetic resonance imaging catheter stress haemodynamics post-Fontan in hypoplastic left heart syndrome. Eur Heart J Cardiovasc Imaging

30. Trusty PM, Restrepo M, Kanter KR et al (2016) A pulsatile hemodynamic evaluation of the commercially available bifurcated Y-graft Fontan modification and comparison with the lateral tunnel and extracardiac conduits. J Thorac Cardiovasc Surg 151:1529-1536

31. Martin MH, Feinstein JA, Chan FP et al (2015) Technical feasibility and intermediate outcomes of using a handcrafted, areapreserving, bifurcated Y-graft modification of the Fontan procedure. J Thorac Cardiovasc Surg 149(239-45):e1

32. Kanski M, Arvidsson PM, Töger J et al (2015) Left ventricular fluid kinetic energy time curves in heart failure from cardiovascular magnetic resonance 4D flow data. J Cardiovasc Magn Reson 17:111

33. Schiavazzi DE, Kung EO, Marsden AL et al (2015) Hemodynamic effects of left pulmonary artery stenosis after superior cavopulmonary connection: a patient-specific multiscale modeling study. J Thorac Cardiovasc Surg 149(689-696):e3

34. Bolger AF, Heiberg E, Karlsson M et al (2007) Transit of blood flow through the human left ventricle mapped by cardiovascular magnetic resonance. J Cardiovasc Magn Reson 9:741-747

35. Mercer-Rosa L (2014) Ventricular function in tetralogy of Fallot: a waste of energy. J Thorac Cardiovasc Surg 19104:19104

36. Penny DJ, Redington AN (1991) Doppler echocardiographic evaluation of pulmonary blood flow after the Fontan operation: the role of the lungs. Br Heart J 66:372-374 